\title{
The estimations of magnetic field fluctuations in turbulent atmospheres
}

\author{
N. A. Silant'ev ${ }^{1,2, \star}$ \\ 1 Instituto Nacional de Astrofísica, Óptica y Electrónica, Apartado Postal 51 y 216, Z.P. 72000, Pue. México \\ 2 Main Astronomical Observatory of Russian Academy of Sciences, 196140 St. Petersburg, Russia \\ Received 18 May 2000 / Accepted 29 November 2000
}

\begin{abstract}
We qualitatively analyze the equations of the magnetohydrodynamics for various quadratic fluctuations of the magnetic field and the vector potential. For the stationary inhomogeneous state, we have established the exact integral relations between the magnetic field fluctuations $\boldsymbol{b}(\boldsymbol{r}, t)$ and the mean magnetic field $\boldsymbol{B}_{0}(\boldsymbol{r}, t)$. We estimate $\alpha \approx(\eta+\beta) \boldsymbol{B}_{0} \cdot\left(\nabla \times \boldsymbol{B}_{0}\right) / B_{0}^{2}$, where $\eta$ and $\beta$ are the ohmic (molecular) and turbulent diffusivities, respectively. The $\alpha$-coefficient describes the enhancement of the mean magnetic field. We found that the exact Seehafer formula $\boldsymbol{B}_{0} \cdot\langle\boldsymbol{u} \times \boldsymbol{b}\rangle=-\eta\langle\boldsymbol{b} \cdot(\nabla \times \boldsymbol{b})\rangle$ is also valid for the locally stationary and homogeneous evolution of magnetic fluctuations ( $\boldsymbol{u}$ is the Euler turbulent velocity). It is shown that the usual $\alpha$-coefficient, more correctly the term $\alpha \boldsymbol{B}_{0} \cdot\left(\nabla \times \boldsymbol{B}_{0}\right)$, presents with opposite signs in equations for $B_{0}^{2}$ and $\left\langle b^{2}\right\rangle$, i.e. the rate of increase of the total magnetic energy does not depend directly on this coefficient. We show that at the moment $t_{b}$ of the maximum of magnetic fluctuations, $\left\langle b^{2}\right\rangle \approx B_{0}^{2}$. The same also holds for the locally homogeneous and stationary regime if the spectrum of the turbulence has a slow decrease in the inertial region of wave numbers. The detailed analysis of two-dimensional turbulence has shown that the Zeldovich estimate $\left\langle b^{2}\right\rangle \approx \beta / \eta B_{0}^{2}$ is valid only at the maximum of the vector potential fluctuations. This estimate is corrected such that at $t_{b}$ it also gives $\left\langle b^{2}\right\rangle \approx B_{0}^{2}$. We also give the approximate expression for the $\alpha$-coefficient which takes into account the back reaction of the mean magnetic field onto the turbulence itself.
\end{abstract}

Key words. magnetic fields - turbulence - stars: magnetic fields - Sun: magnetic fields

\section{Introduction}

Because of the complexity of the magnetic field behaviour in turbulent media, one uses approximate methods to describe these phenomena. Most frequently, especially in the magnetic dynamo theory, the diffusion approximation is used. In this approximation, by the averaging the basic induction equation

$\frac{\partial \boldsymbol{B}}{\partial t}=\nabla \times\left(\boldsymbol{U}_{0} \times \boldsymbol{B}\right)+\nabla \times(\boldsymbol{u} \times \boldsymbol{B})+\eta \nabla^{2} \boldsymbol{B}$,

one assumes that

$\boldsymbol{F}_{0} \equiv\langle\boldsymbol{u} \times \boldsymbol{b}\rangle \simeq \alpha(\boldsymbol{r}, t) \boldsymbol{B}_{0}(\boldsymbol{r}, t)-\beta(\boldsymbol{r}, t) \nabla \times \boldsymbol{B}_{0}(\boldsymbol{r}, t)$

Here $\boldsymbol{U}_{0}$ and $\boldsymbol{u}$ are regular and turbulent Euler velocities of the medium, $\eta$ is the ohmic (molecular) diffusivity, the magnetic field $\boldsymbol{B}(\boldsymbol{r}, t)=\boldsymbol{B}_{0}(\boldsymbol{r}, t)+\boldsymbol{b}(\boldsymbol{r}, t)$ is the sum of the mean value $\langle\boldsymbol{B}\rangle \equiv \boldsymbol{B}_{0}$ and the fluctuating part $\boldsymbol{b}$, with $\langle\boldsymbol{b}\rangle=0$. We represent all stochastic values as a sum of the mean and fluctuating components, for example, the vector potential $\boldsymbol{A}(\boldsymbol{r}, t)=\boldsymbol{A}_{0}(\boldsymbol{r}, t)+\boldsymbol{a}(\boldsymbol{r}, t)$, with $\langle\boldsymbol{a}\rangle=0$, etc.

Thus, the mean electromotive force $\boldsymbol{F}_{0}$ in the diffusion approximation consists of two terms. The term with

\footnotetext{
* e-mail: silant@inaoep.mx
}

$\alpha(\boldsymbol{r}, t)$ describes the turbulent enhancement of the mean magnetic field ( $\alpha$-effect). The $\alpha(\boldsymbol{r}, t)$ - function is pseudoscalar and exists if the turbulence is mirror asymmetric. The value $\beta(\boldsymbol{r}, t)$ is the turbulent diffusivity.

Expression (2) is valid if the mean magnetic field $\boldsymbol{B}_{0}(\boldsymbol{r}, t)$ is a smooth function at the characteristic scale $R_{0}$ and characteristic time $\tau_{0}$ of the turbulent velocity correlations. If the turbulent "turnover time" $t_{0} \equiv R_{0} / u_{0} \ll \tau_{0}$, then the smoothness of $\boldsymbol{B}_{0}$ at the time interval $t_{0}$ is sufficient. The value $u_{0}$ is the characteristic turbulent velocity, $u_{0}^{2}=\left\langle\boldsymbol{u}^{2}(\boldsymbol{r}, t)\right\rangle$. Formula (2) has been derived by the assumption of local isotropy of the turbulent velocity field. In the general case (see Krause \& Rädler 1980; Moffatt 1978 ), instead of $\alpha$ and $\beta$, one needs to use some pseudotensor of the second rank and a tensor of the third rank, respectively. Previously the simple representation (2) has been used, as it is now, due to its simplicity. Thus, for the diffusion approximation one has:

$$
\begin{aligned}
\frac{\partial \boldsymbol{B}_{0}}{\partial t}= & \nabla \times\left(\boldsymbol{U}_{0} \times \boldsymbol{B}_{0}(\boldsymbol{r}, t)\right)+\nabla \times \alpha(\boldsymbol{r}, t) \boldsymbol{B}_{0}(\boldsymbol{r}, t) \\
& +\nabla \times[\eta+\beta(\boldsymbol{r}, t)] \nabla \times \boldsymbol{B}_{0}(\boldsymbol{r}, t) .
\end{aligned}
$$

Usually one uses the estimates (Moffatt 1978; Krause \& Rädler 1980; Weiss 1994):

$\alpha \approx u_{0}, \quad \beta \approx u_{0} R_{0}$ 
More detailed estimates of these coefficients are presented in other papers (Silant'ev 1997a,b). It is shown that the stationary values $\alpha$ and $\beta$ depend strongly on the turbulent Strouhal number $\xi_{0}=u_{0} \tau_{0} / R_{0}$. For $\xi_{0} \ll 1$,

$\alpha \approx-u_{0} \xi_{0} a \tau_{\mathrm{h}} / 3 \tau_{0}$

$$
\beta \approx u_{0} R_{0} \xi_{0} / 3 \simeq u_{0}^{2} \tau_{0} / 3
$$

where $a=H_{0} / u_{0}^{2} p_{0}$ is the degree of turbulent helicity, $a \leq 1, H_{0}=\langle\boldsymbol{u} \cdot(\nabla \times \boldsymbol{u})\rangle$ is the mean helicity, $p_{0} \simeq 1 / R_{0}$ and $p_{\mathrm{h}}$ are characteristic wave numbers corresponding to spectra of turbulent energy $E(p, \tau)$ and helicity $E_{\mathrm{h}}(p, \tau)$. These spectra are determined by the relations:

$$
\begin{aligned}
& \langle\boldsymbol{u}(\boldsymbol{r}, t) \cdot \boldsymbol{u}(\boldsymbol{r}, t+\tau)\rangle=\int_{0}^{\infty} \mathrm{d} p E(p, \tau), \\
& \left\langle\boldsymbol{u}(\boldsymbol{r}, t) \cdot(\nabla \times \boldsymbol{u}(\boldsymbol{r}, t+\tau)\rangle=\int_{0}^{\infty} \mathrm{d} p E_{\mathrm{h}}(p, \tau) .\right.
\end{aligned}
$$

They are characterized by the parameters $u_{0}^{2}, p_{0}, \tau_{0}$ and $H_{0}, p_{\mathrm{h}}, \tau_{\mathrm{h}}$, respectively. For $\xi_{0} \gg 1$ :

$\alpha \approx-H_{0} / u_{0} p_{\mathrm{h}} \simeq-a p_{0} u_{0} / p_{\mathrm{h}}, \quad \beta \approx u_{0} / p_{0} \sqrt{3}$.

Estimation (4) corresponds to $\xi_{0} \gg 1$ and $a=1, p_{0}=p_{\mathrm{h}}$. We note that $\alpha \leq u_{0}$.

When the magnetic energy $B^{2} / 8 \pi$ is comparable with the energy of turbulent motions, $\rho u^{2} / 2$, the structure of the turbulence changes under the action of the Lorentz force (where $\rho$ is the density of the medium). In this case, all parameters depend on the value of the mean magnetic field $B_{0}$. For this reason, we use in the estimates the observable values of all parameters, corresponding to real turbulence. Rüdiger \& Kitchatinov (1993) and Kichatinov et al. (1994), using a numerical solution of Navier-Stokes and induction equations, have shown that the parameters $\alpha$ and $\beta$ are suppressed by large magnetic fields.

The estimates of the magnetic field fluctuations are of special interest after the derivation of the formula:

$\alpha=-\eta\langle\boldsymbol{b} \cdot(\nabla \times \boldsymbol{b})\rangle / B_{0}^{2}$.

This formula was derived in a rather unsatisfactory fashion in the papers (Gruzinov \& Diamond 1994; Vainshtein 1998). This formula is the particular case of the exact Seehafer $(1994,1996)$ expression

$\langle\boldsymbol{u} \times \boldsymbol{b}\rangle \cdot \boldsymbol{B}_{0} \equiv \boldsymbol{F}_{0} \cdot \boldsymbol{B}_{0}=-\eta\langle\boldsymbol{b} \cdot(\nabla \times \boldsymbol{b})\rangle$,

and corresponds to the stationary and homogeneous state of the magnetic field and turbulence (in this case $\langle\boldsymbol{u} \times \boldsymbol{b}\rangle=$ $\alpha \boldsymbol{B}_{0}$ and we obtain (9)). Note that we do not use the inconvenient notion of Seehafer, $\boldsymbol{F}_{0} \cdot \boldsymbol{B}_{0} \equiv \alpha B_{0}^{2}$ for the general case of inhomogeneous turbulence. His notion joins two different effects - turbulent diffusion and enhancement of magnetic field - as one generalized $\alpha$-effect.

Usually, one can conclude from (9) that the value $\alpha \approx u_{0}$, needed for the action of the magnetic dynamo, corresponds to a very large level of magnetic fluctuations, $\left\langle b^{2}\right\rangle \equiv b_{0}^{2} \simeq R_{\mathrm{m}} B_{0}^{2}$, where $R_{\mathrm{m}} \simeq \beta / \eta$ is the magnetic Reynolds number. In cosmic conditions the values of $R_{\mathrm{m}}$ are huge, $\sim 10^{9}-10^{14}$. The observations do not show the existence of very large magnetic fluctuations. For this reason, many authors (see, for example, Vainshtein \& Rosner 1991; Vainshtein \& Cattaneo 1992; Gruzinov \& Diamond 1994) consider that the usual $\alpha$-effect does not occur in cosmic objects.

Here we improve this commonly - accepted estimate. Indeed, from Eq. (10) we obtain the estimate $b_{0} \sim\left(u_{0} l_{0} / \eta\right) B_{0}$, after which, Eq. (9) gives rise to $\alpha \sim$ $\left(u_{0} l_{0} / \eta\right) u_{0}$. Here $l_{0}$ is the characteristic scale of the variation of magnetic field fluctuations. For the turbulent spectra with a slow decrease (as in Kolmogorov's and Kraichnan's spectra), this parameter is near the scale of the viscous dissipation and $u_{0} l_{0} \sim \eta$. Thus, we do not need huge magnetic fluctuations to support the $\alpha$-dynamo mechanism. It should be noted that these estimates are the maximum ones, because they do not take into account the angular dependence of the vector formulae. Particularly, they do not depend on the degree of the turbulent helicity. We know that the absence of helicity gives rise to $\alpha \equiv 0$.

There exists another reason why the estimates of the magnetic field fluctuations are of the interest. If the level of fluctuations is high, then the fluctuation alone can change the character of turbulent motions, and not the mean magnetic field itself (Vainshtein \& Rosner 1991; Vainshtein \& Cattaneo 1992).

Zeldovich (1957), for the case of two-dimensional turbulence, has shown the existence of very large magnetic fluctuations, of the order $\left\langle b^{2}\right\rangle \simeq R_{\mathrm{m}} B_{0}^{2}$. According to his scenario, two-dimensional turbulent motions during the turbulent mixing time $t_{\beta} \approx L_{0}^{2} / \beta$ transform the initial magnetic field $\boldsymbol{B}_{0}(\boldsymbol{r})$ into a purely fluctuating field with the maximum value at $t \approx t_{\beta}$ being equal to $\left\langle b^{2}\right\rangle \simeq R_{\mathrm{m}} B_{0}^{2}(\boldsymbol{r})$ (here $L_{0}$ is the scale of the variations of $\left.\boldsymbol{B}_{0}(\boldsymbol{r})\right)$. According to Zeldovich, these huge fluctuations also eventually disappear in the characteristic time $t_{\beta}$. The Zeldovich estimate also came into use for real 3D-turbulence (Vainshtein \& Rosner 1991; Vainshtein \& Cattaneo 1992; Gruzinov \& Diamond 1994).

The goal of the present paper is to show that this level of magnetic fluctuations is highly overestimated. Our improved estimates demonstrate that the usual $\alpha$-effect can be used to explane the existence of cosmic magnetic fields.

To derive new estimates, we used the technique of Seehafer (1996). We analyzed pairs of coupled equations for $\boldsymbol{A}_{0} \cdot \boldsymbol{B}_{0}$ and $\langle\boldsymbol{a} \cdot \boldsymbol{b}\rangle, \boldsymbol{A}_{0}^{2}$ and $\left\langle\boldsymbol{a}^{2}\right\rangle, \boldsymbol{B}_{0}^{2}$ and $\left\langle\boldsymbol{b}^{2}\right\rangle$. With these equations, the fluctuation dynamics can be traced more clearly than using the means $\langle\boldsymbol{A} \cdot \boldsymbol{B}\rangle,\left\langle\boldsymbol{A}^{2}\right\rangle$ and $\left\langle\boldsymbol{B}^{2}\right\rangle$. Following Zeldovich (1957), the analysis will be carried out on the volume-integrated quantities, so that the flux terms containing divergences disappear (due to the divergence theorem). These estimations correspond to real boundary conditions occurring when all the fields tend to zero, far from the considered volume.

\section{Equations for magnetic helicity}

Seehafer $(1994,1996)$ has derived a system of two exact equations for the evolution of magnetic helicity 
$H_{\mathrm{m}} \equiv\langle\boldsymbol{A} \cdot \boldsymbol{B}\rangle=\boldsymbol{A}_{0} \cdot \boldsymbol{B}_{0}+\langle\boldsymbol{a} \cdot \boldsymbol{b}\rangle:$

$$
\begin{aligned}
\frac{\partial \boldsymbol{A}_{0} \cdot \boldsymbol{B}_{0}}{\partial t}= & -2 \eta \boldsymbol{B}_{0} \cdot\left(\nabla \times \boldsymbol{B}_{0}\right)+2 \boldsymbol{B}_{0} \cdot \boldsymbol{F}_{0} \\
& -\operatorname{div}\left(c \boldsymbol{E}_{0} \times \boldsymbol{A}_{0}-c \boldsymbol{B}_{0} \varphi_{0}\right),
\end{aligned}
$$

$$
\begin{aligned}
\frac{\partial\langle\boldsymbol{a} \cdot \boldsymbol{b}\rangle}{\partial t}= & -2 \eta\langle\boldsymbol{b} \cdot(\nabla \times \boldsymbol{b})\rangle-2 \boldsymbol{B}_{0} \cdot \boldsymbol{F}_{0} \\
& -\operatorname{div}\langle c \boldsymbol{e} \times \boldsymbol{a}-c \boldsymbol{b} \varphi\rangle .
\end{aligned}
$$

Here, the mean electromotive force is $\boldsymbol{F}_{0}=\langle\boldsymbol{u} \times \boldsymbol{b}\rangle, \boldsymbol{E}=$ $\boldsymbol{E}_{0}+\boldsymbol{e}(\langle\boldsymbol{e}\rangle=0)$ is the electric field, $c$ is the speed of light, $\varphi_{0}$ and $\varphi$ are the regular and stochastic components of the scalar potential. One of the Maxwell equations reads:

$\frac{\partial \boldsymbol{B}}{\partial t}=-c \nabla \times \boldsymbol{E}$.

The expression for the electric field (Landau \& Lifshitz 1960; Roberts 1967) is:

$c \boldsymbol{E}=\eta \nabla \times \boldsymbol{B}-\boldsymbol{u} \times \boldsymbol{B}-\boldsymbol{U}_{0} \times \boldsymbol{B}$.

Substituting Eq. (14) into Eq. (13) gives the basic induction Eq. (1). Equations (11) and (12) can easily be derived from (1), (13), (14) and the relation $\partial \boldsymbol{A} / \partial t=$ $-c \boldsymbol{E}+c \nabla \varphi$. The vector identity $\operatorname{div} \boldsymbol{A} \times \boldsymbol{B}=\boldsymbol{B} \cdot(\nabla \times$ $\boldsymbol{A})-\boldsymbol{A} \cdot(\nabla \times \boldsymbol{B})$ must also be used.

It is seen from Eqs. (11) and (12) that the $\alpha$-effect (more precisely, the term $2 \boldsymbol{B}_{0} \cdot \boldsymbol{F}_{0}$ ) enhances (or reduces) the large-scale magnetic helicity and reduces (or enhances) to the same extent its small-scale fluctuating component. The total mean helicity $H_{\mathrm{m}}=\langle\boldsymbol{A} \cdot \boldsymbol{B}\rangle$ does not depend on the electromotive force. This statement is the main conclusion of Seehafer's papers. We stress that in considering the influence of the $\alpha$-effect on the evolution of the magnetic fluctuations, we must preserve this zero balance exactly. It means that both Eqs. (11) and (12) are equally important to such a consideration. In reality, it is more convenient to use the Maxwell equation $\partial \boldsymbol{B}_{0} / \partial t=-c \nabla \times \boldsymbol{E}_{0}$ instead of (11).

Let us consider a steady-state magnetic field in a turbulent medium. In this case $\partial\langle\boldsymbol{a} \cdot \boldsymbol{b}\rangle / \partial t=0, \boldsymbol{B}_{0}(\boldsymbol{r}, t)=$ $\boldsymbol{B}_{0}(\boldsymbol{r})$, and, hence, $c \nabla \times \boldsymbol{E}_{0}=-\partial \boldsymbol{B}_{0} / \partial t=0$, i.e. $\boldsymbol{E}_{0}=\nabla \varphi_{0}(\boldsymbol{r})$. Averaging Eq. (14) then gives:

$\boldsymbol{F}_{0}=\eta \nabla \times \boldsymbol{B}_{0}(\boldsymbol{r})-\boldsymbol{U}_{0} \times \boldsymbol{B}_{0}-c \nabla \varphi_{0}(\boldsymbol{r})$.

Substitution of Eq. (15) into Eq. (11) transforms the latter to the identity. After substituting Eq. (15) into Eq. (12) and volume integration, we find:

$\int \mathrm{d} V\left[\langle\boldsymbol{b} \cdot(\nabla \times \boldsymbol{b})\rangle+\boldsymbol{B}_{0} \cdot\left(\nabla \times \boldsymbol{B}_{0}\right)\right]=0$.

The term $\boldsymbol{B}_{0}(\boldsymbol{r}) \nabla \varphi_{0}(\boldsymbol{r})=\operatorname{div} \boldsymbol{B}_{0}(\boldsymbol{r}) \varphi_{0}(\boldsymbol{r})$ disappears after applying the divergence theorem.

The scalar product $\boldsymbol{F}_{0} \cdot \boldsymbol{B}_{0}$ follows from Eq. (15). The volume integration of this quantity gives rise to the second exact integral relationship:

$\int \mathrm{d} V\left[\boldsymbol{F}_{0} \cdot \boldsymbol{B}_{0}-\eta \boldsymbol{B}_{0} \cdot\left(\nabla \times \boldsymbol{B}_{0}\right)\right]=0$.
The combination of (16) and (17) gives the third exact relation:

$\int \mathrm{d} V\left[\boldsymbol{F}_{0} \cdot \boldsymbol{B}_{0}+\eta\langle\boldsymbol{b} \cdot(\nabla \times \boldsymbol{b})\rangle\right]=0$.

These exact integral relations also follow directly from basic scalar Eqs. (11) and (12). Our derivation is based on the vector expression (15) which will be used later to derive the estimate of the $\alpha$-coefficient.

It should be stressed that these exact integral relations were obtained without using the Navier-Stokes equation, i.e. they automatically take into account the back reaction of the magnetic field onto the structure of the turbulent velocity field. The Lorentz force acts on the velocity field and vice versa. For this reason, the stationary state of the magnetic field exists only if the velocity field is also stationary.

Of course, to support the system in a stationary state, we need some sources of mechanical or thermal energy. However, the source terms exist only in the Navier Stokes equation; the magnetic induction Eq. (1) is free of such terms. The stationary state of the magnetic field may also be provided by some appropriate boundary conditions (Peckover \& Weiss 1978). The behaviour of the magnetic field is quite different for various boundary conditions. Our exact integral relationships correspond to the usual astrophysical situation when the magnetic and electric fields tend to zero beyond the considered volume.

The exact integral relationships (16), (17) and (18) can be used as additional criteria for the correctness of numerical calculations of the evolution of a magnetic field on its way to the stationary state.

An unknown angular dependence of the vector expressions in the integrands of these relationships does not allow us to derive the estimates between some local characteristic values of the involved quantities. It seems that the only exception is the case when the integrands consist of quadratic values (see Eq. (35) and the case of twodimensional turbulence). On the contrary, local estimates can be derived from the differential equations. In this case, the sine and cosine of unknown angles may be considered as unknown numerical coefficients. For this reason, we give here the estimate of the $\alpha$-coefficient following from the vector differential relation (15).

Making use of the well-known (Morse \& Feshbach 1953) expression for a curl-free field through its divergence, the following explicit expression can be written from Eq. (15) for the potential $\varphi_{0}(\boldsymbol{r})$ :

$c \varphi_{0}(\boldsymbol{r})=\operatorname{div} \int \mathrm{d} V^{\prime}\left[\langle\boldsymbol{u} \times \boldsymbol{b}\rangle+\boldsymbol{U}_{0} \times \boldsymbol{B}_{0}\right]\left|\boldsymbol{r}-\boldsymbol{r}^{\prime}\right|^{-1} / 4 \pi$

This expression is purely formal, because the integrand contains unknown functions. The diffusion approximation (2) implies that $\boldsymbol{B}_{0}(\boldsymbol{r})$ and $\boldsymbol{U}_{0}(\boldsymbol{r})$ are smooth on the turbulence scale, $\sim R_{0}$. In this case, the term $\operatorname{div} \boldsymbol{B}_{0}(\boldsymbol{r}) \varphi_{0}(\boldsymbol{r})$, containing double differentiation of a 
smooth function, is smaller than the first term in Eq. (15). This yields the following estimate:

$\alpha(\boldsymbol{r}) \approx(\eta+\beta(\boldsymbol{r})) \boldsymbol{B}_{0}(\boldsymbol{r}) \cdot\left(\nabla \times \boldsymbol{B}_{0}(\boldsymbol{r})\right) / B_{0}^{2}$.

For isotropic turbulence, this relation qualitatively coincides with the results of numerical calculations (see (5) and (8)).

The Seehafer relation (10) follows from Eq. (12) for stationary and homogeneous turbulence. The discussion of this relationship was given in the Introduction. In the next section, we show that this relationship is also valid for the case of locally homogeneous and stationary magnetic fluctuations.

\section{Qualitative analysis of the equations for magnetic energy}

It is of interest to analyze the system of equations for the values $B_{0}^{2}$ and $\left\langle b^{2}\right\rangle$. Here and in that which follows, we take $\boldsymbol{U}_{0}=0$. First, we give the equations in their exact form:

$$
\begin{aligned}
\frac{\partial B_{0}^{2}}{\partial t}= & -2 \eta\left(\nabla \times \boldsymbol{B}_{0}\right)^{2}+2 \boldsymbol{B}_{0} \cdot\left(\nabla \times \boldsymbol{F}_{0}\right)+\eta \nabla^{2} B_{0}^{2} \\
& -2 \eta \operatorname{div}\left(\boldsymbol{B}_{0} \cdot \nabla\right) \boldsymbol{B}_{0},
\end{aligned}
$$

$$
\begin{aligned}
\frac{\partial\left\langle b^{2}\right\rangle}{\partial t}= & -2 \eta\left\langle(\nabla \times \boldsymbol{b})^{2}\right\rangle+2\langle\boldsymbol{b} \cdot(\nabla \times(\boldsymbol{u} \times \boldsymbol{B}))\rangle \\
& +\eta \nabla^{2}\left\langle b^{2}\right\rangle-2 \eta \operatorname{div}(\boldsymbol{b} \cdot \nabla) \boldsymbol{b} .
\end{aligned}
$$

The dynamics of the magnetic energy evolution is more representative in the diffusion approximation when Eqs. (21) and (22) acquire the form:

$$
\begin{aligned}
\frac{\partial B_{0}^{2}}{\partial t}= & -2(\eta+\beta)\left(\nabla \times \boldsymbol{B}_{0}\right)^{2}+2 \alpha \boldsymbol{B}_{0} \cdot\left(\nabla \times \boldsymbol{B}_{0}\right) \\
& +(\eta+\beta) \nabla^{2} B_{0}^{2}-2(\eta+\beta) \operatorname{div}\left(\boldsymbol{B}_{0} \cdot \nabla\right) \boldsymbol{B}_{0}, \\
\frac{\partial\left\langle b^{2}\right\rangle}{\partial t}= & -2 \eta\left\langle(\nabla \times \boldsymbol{b})^{2}\right\rangle+2(\beta+2 \sigma-\delta)\left(\nabla \times \boldsymbol{B}_{0}\right)^{2} \\
& -2 \alpha \boldsymbol{B}_{0} \cdot\left(\nabla \times \boldsymbol{B}_{0}\right)++2 \gamma B_{0}^{2} \\
& +2\langle\boldsymbol{b} \cdot(\nabla \times(\boldsymbol{u} \times \boldsymbol{b}))\rangle+\left(\delta-\sigma-\beta_{1}\right) \nabla^{2} B_{0}^{2} \\
& +\eta \nabla^{2}\left\langle b^{2}\right\rangle+2\left(3 \sigma+\beta-\beta_{1}-\delta\right) \operatorname{div}\left(\boldsymbol{B}_{0} \cdot \nabla\right) \boldsymbol{B}_{0} \\
& -2 \eta \operatorname{div}(\boldsymbol{b} \cdot \nabla) \boldsymbol{b} .
\end{aligned}
$$

The transport coefficients $\alpha, \beta, \gamma, \sigma$ and $\delta$ are calculated in the nonlocal representation when

$b_{i}(\boldsymbol{r}, t)=$

$\int \mathrm{d} \boldsymbol{r}^{\prime} \int_{0}^{t} \mathrm{~d} t^{\prime} G_{i j}(\boldsymbol{R}, \tau)\left[\nabla^{\prime} \times\left(\boldsymbol{u}\left(\boldsymbol{r}^{\prime}, t^{\prime}\right) \times \boldsymbol{B}_{0}\left(\boldsymbol{r}^{\prime}, t^{\prime}\right)\right)\right]_{j}$.

Here, $G_{i j}$ is the averaged Green tensor of Eq. (1) for homogeneous, isotropic and stationary turbulence, $\boldsymbol{R}=\boldsymbol{r}-\boldsymbol{r}^{\prime}$, $\tau=t-t^{\prime}$. It is known (see Kraichnan 1976; Silant'ev $1997 \mathrm{a}, \mathrm{b})$ that, to a reasonable approximation, the $G_{i j}$ satisfies the nonlinear direct interaction approximation equation (DIA-equation). Silant'ev (1997a,b) first obtained the exact numerical solution of this tensor DIAequation and demonstrated its effectiveness by the calculation of the turbulent transport coefficients. The results of these calculations were discussed in the Introduction.

The Green tensor, $G_{i j}$, has the form:

$G_{i j}=\delta_{i j} G_{0}(R, \tau)+e_{i j k} R_{k} G_{1}(R, \tau)$.

Here, $e_{\mathrm{pts}}$ is the unit antisymmetric pseudotensor $\left(e_{x y z}=\right.$ $-e_{y x z}=-e_{x z y}=1$, etc.).

The spatial Fourier transform of $G_{i j}$ has a similar form:

$g_{i j}(\boldsymbol{p}, \tau)=\delta_{i j} g_{0}(p, \tau)+i e_{i j k} p_{k} g_{1}(p, \tau)$.

The correlator of turbulent velocities is assumed to be of the form:

$$
\begin{aligned}
\left\langle u_{i}(\boldsymbol{r}, t) u_{j}\left(\boldsymbol{r}^{\prime}, t^{\prime}\right)\right\rangle \equiv & B_{i j}(\boldsymbol{R}, \tau)= \\
& B_{i j}^{(s)}(\boldsymbol{R}, \tau)+e_{i j k} R_{k} D(R, \tau) .
\end{aligned}
$$

The function $D(R, \tau)$ describes the helicity of turbulent motions, $B_{i j}^{(s)}$ denotes the symmetric components of the correlation tensor.

Now we give the explicit formulae for the transport coefficients:

$$
\begin{aligned}
\alpha(t)= & -\frac{1}{3} \int_{0}^{\infty} \mathrm{d} p \int_{0}^{t} \mathrm{~d} \tau\left[g_{0}(p, \tau) E_{\mathrm{h}}(p, \tau)\right. \\
& \left.-p^{2} g_{1}(p, \tau) E(p, \tau)\right],
\end{aligned}
$$

$\beta(t)=\frac{1}{3} \int_{0}^{\infty} \mathrm{d} p \int_{0}^{t} \mathrm{~d} \tau\left[g_{0}(p, \tau) E(p, \tau)\right.$ $\left.-g_{1}(p, \tau) E_{\mathrm{h}}(p, \tau)\right]$

$$
\begin{aligned}
\gamma(t)= & \frac{1}{3} \int_{0}^{\infty} \mathrm{d} p \int_{0}^{t} \mathrm{~d} \tau p^{2}\left[g_{0}(p, \tau) E(p, \tau)\right. \\
& \left.-g_{1}(p, \tau) E_{\mathrm{h}}(p, \tau)\right]
\end{aligned}
$$

$\sigma=\frac{1}{30} \int \mathrm{d} \boldsymbol{R} \int_{0}^{t} \mathrm{~d} \tau\left[G_{0}(\boldsymbol{R} \cdot \nabla) B_{i i}+2 G_{1} R^{2}(\boldsymbol{R} \cdot \nabla) D\right]$,

$\beta_{1}=\frac{1}{3} \int \mathrm{d} \boldsymbol{R} \int_{0}^{t} \mathrm{~d} \tau G_{1} R^{2} D$

$\delta=-\frac{1}{18} \int \mathrm{d} \boldsymbol{R} \int_{0}^{t} \mathrm{~d} \tau R^{2}\left[G_{0} \nabla^{2} B_{i i}+2 G_{1} R_{i} \nabla^{2}\left(R_{i} D\right)\right]$.

Here, $E(p, \tau)$ and $E_{\mathrm{h}}(p, \tau)$ are the spectra of turbulence and the turbulent helicity, correspondingly (see (6) and (7)).

The term $\langle\boldsymbol{b} \cdot(\nabla \times(\boldsymbol{u} \times \boldsymbol{b}))\rangle$ contains the velocity correlators of the third and higher orders. We do not present here its very complicated explicit form. It is clear that this expression does not contain terms such as $\alpha, \beta, \gamma, \sigma$ and $\delta$. 
The terms with $\nabla^{2}$ and div disappear upon volume integration, and they will be ignored in the subsequent estimates. First of all, we see that the usual $\alpha$-effect enhances the energy of large-scale magnetic fields and reduces, to the same degree, the energy of magnetic fluctuations. This does not allow magnetic fluctuations to be very large. In the stationary state, the balance between the enhancement of the large-scale component and its weakening is mainly provided by the self-consistency of the coefficients $\alpha$ and $\beta: \alpha \approx \beta / L_{0}$. The same estimate follows from Eq. (20). Here, $L_{0}$ is the scale of variation of the mean magnetic field $\boldsymbol{B}_{0}$. In the estimations we shall use its minimum value $L_{0} \approx R_{0}$.

Thus, we see that the sum of Eqs. (23) and (24), describing the evolution of the total magnetic energy, $\left\langle B^{2}\right\rangle$, does not depend on the terms $\alpha \boldsymbol{B}_{0} \cdot\left(\nabla \times \boldsymbol{B}_{0}\right)$ and $\beta\left(\nabla \times \boldsymbol{B}_{0}\right)^{2}$. This statement is exact, as follows from the derivation of the diffusion equation for $\left\langle B^{2}\right\rangle$ using the Lagrangian representation. This derivation uses the known (see Roberts 1967; Parker 1980; Moffatt 1978) exact solution of the induction Eq. (1) for the case $\eta=0$. The technique of such derivation is presented in the paper (Moffatt 1974). Omitting the details of the calculations, we give here only the final equation:

$\frac{\partial\left\langle B^{2}\right\rangle}{\partial t}=2 \gamma B_{0}^{2}+\mu \nabla^{2} B_{0}^{2}+2 \eta\left\langle\boldsymbol{B} \cdot \nabla^{2} \boldsymbol{B}\right\rangle \cdot$

The coefficients $\gamma$ and $\mu$ have the form:

$\gamma=\frac{1}{3}\left\langle\frac{\partial X_{i}}{\partial a_{j}} \frac{\partial v_{i}}{\partial a_{j}}\right\rangle$

$\mu=\frac{1}{3}\langle\boldsymbol{v} \cdot \boldsymbol{X}\rangle+\frac{1}{9}\left\langle\boldsymbol{v} \cdot \boldsymbol{X}\left(\frac{\partial X_{i}}{\partial a_{j}}\right)^{2}\right\rangle+\frac{1}{9}\left\langle X^{2} \frac{\partial X_{i}}{\partial a_{j}} \frac{\partial v_{i}}{\partial a_{j}}\right\rangle$

The radius-vector $\boldsymbol{r}$ of the point of the observation is connected with the Lagrange velocity $\boldsymbol{v}(\boldsymbol{a}, t)$ by the relation (see Roberts 1967):

$\boldsymbol{r}=\boldsymbol{a}+\boldsymbol{X}(\boldsymbol{a}, t) \equiv \boldsymbol{a}+\int_{0}^{t} \mathrm{~d} \tau \boldsymbol{v}(\boldsymbol{a}, \tau)$.

Here, $\boldsymbol{a}$ is the initial position of a liquid particle. We also note that the identities $2 \boldsymbol{B} \cdot \nabla^{2} \boldsymbol{B}=\nabla^{2} B^{2}-2\left(\nabla_{i} B_{j}\right)^{2}$ and $\left(\nabla_{i} B_{j}\right)^{2}=(\nabla \times \boldsymbol{B})^{2}+\operatorname{div}(\boldsymbol{B} \cdot \nabla) \boldsymbol{B}$ were used by the derivation of Eqs. (23) and (24).

The absence of the terms with $\alpha$ and $\beta$ in Eq. (35) does not mean that the total magnetic energy is independent of the $\alpha$-effect and turbulent diffusion. This equation is not closed. We need to know the energy of the mean magnetic field $B_{0}^{2}$ to calculate the total magnetic energy. Thus, the scenario of the evolution of the initial large-scale magnetic field $\boldsymbol{B}_{0}$ appears as follows: first the turbulent deformations and rotations of a fluid (the term with $\gamma$ in (24)), and the turbulent diffusion, which is mainly due to convective motions (term with $\beta$ in (24)), transform the large-scale magnetic field into a small-scale, fluctuating field. The evolution of values $B_{0}^{2}$ and $\left\langle b^{2}\right\rangle$ depends on the relative value of the $\alpha$-coefficient compared to the turbulent diffusivity $\beta$.

If the $\alpha$-effect is absent, or small, the large-scale magnetic energy decreases with the characteristic time $t_{\beta} \approx$ $R_{0}^{2} / \beta$. The fluctuations $\left\langle b^{2}\right\rangle$, according to Eq. (24), first grow, and at the time $t_{b}$ acquire its maximum value, after which they decrease. The time $t_{b}$ and the characteristic time of the decrease of $\left\langle b^{2}\right\rangle$ depend on another characteristic time, $t_{\eta} \approx l_{0}^{2} / \eta$. If $t_{\eta} \gg t_{\beta}$, one has $t_{b} \sim t_{\beta}$ and the fluctuations decrease with characteristic time $t_{\eta}$. This corresponds to $l_{0} \sim R_{0}$, i.e. the spectrum of turbulence $E(p)$ has a sharp peak near $p \approx 1 / R_{0}$ and then rapidly decreases at high wave numbers. Large values of $l_{0}$ provide very slow disappearance of the magnetic fluctuations due to the action of the ohmic losses. In the opposite case $t_{\eta} \ll t_{\beta}$, or $l_{0} u_{0} \sim \eta$, one has $t_{b} \sim t_{\eta}$ and the fluctuations decrease with the characteristic time $t_{\beta}$. This case corresponds to the spectra of turbulence with a slow decrease in the inertial range of the wave numbers, such as the Kolmogorov and Kraichnan spectra of turbulence. It appears that such spectra are common in nature.

If the $\alpha$-effect is not suppressed by turbulent diffusion, then both the mean magnetic field $\boldsymbol{B}_{0}$ and the fluctuating magnetic energy tend to some stationary state. The characteristic time of the establishment of this state is of the order of $t_{\beta}$ for the mean magnetic field. If $t_{\eta} \gg t_{\beta}$, magnetic fluctuations tends to the stationary state with the characteristic time $t_{\eta}$. For the opposite case, $t_{\eta} \ll t_{\beta}$, the magnetic fluctuations acquire the stationary state simultaneously with the mean magnetic field.

The evolution of the vector potential, $\boldsymbol{A}=\boldsymbol{A}_{0}+\boldsymbol{a}$, and magnetic helicity, $\boldsymbol{A} \cdot \boldsymbol{B}$, is qualitatively equivalent. For the case $t_{\eta} \ll t_{\beta}$, the term with the time derivative in Eq. (12) is of the order $\langle\boldsymbol{a} \cdot \boldsymbol{b}\rangle / t_{\beta}$, and is much smaller than the first term in right-hand part of this equation. In this case, the divergence term in (12) is also small, of the order $\left(l_{0} t_{\eta}\right) /\left(R_{0} t_{\beta}\right)$, compared to the term $2 \eta\langle\boldsymbol{b} \cdot(\nabla \times$ $\boldsymbol{b})\rangle$. This case corresponds to the local stationarity and homogeneity of magnetic fluctuations. Thus, the Seehafer formula (10) is also valid for the case $t_{\eta} \ll t_{\beta}$. The mean magnetic field can depend on time and possesses largescale inhomogeneity in this case. In such a case, we can describe the Seehafer formula as

$\alpha B_{0}^{2}=\beta \boldsymbol{B}_{0} \cdot\left(\nabla \times \boldsymbol{B}_{0}\right)-\eta\langle\boldsymbol{b} \cdot(\nabla \times \boldsymbol{b})\rangle \cdot$

The estimation of the fluctuations $\left\langle b^{2}\right\rangle$ will be obtained for the moment when $\partial\left\langle b^{2}\right\rangle / \partial t=0$. This corresponds to the possible maximum of fluctuations (in the absence of the $\alpha-$ effect this maximum certainly exists) or to the stationary state. For the case $t_{\eta} \ll t_{\beta}$ this derivative can be omitted for all the times greater then $t_{\eta}$. We note that the term $\eta \nabla^{2}\left\langle b^{2}\right\rangle$ in Eq. (22) is $\left(R_{0} / l_{0}\right)^{2}$ times smaller than the first 
term and can be omitted. Thus, we have to evaluate the expression:

$$
\begin{aligned}
-2 \eta\left\langle\left(\nabla_{i} b_{j}\right)^{2}\right\rangle & +2\left\langle\boldsymbol{b} \cdot\left(\nabla \times\left(\boldsymbol{u} \times \boldsymbol{B}_{0}\right)\right)\right\rangle \\
& +2\langle\boldsymbol{b} \cdot(\nabla \times(\boldsymbol{u} \times \boldsymbol{b}))\rangle=0 .
\end{aligned}
$$

It is evident that the third term is much larger than the first (they are of the orders $b^{2} u_{0} / l_{0}$ and $b^{2} \eta / l_{0}^{2}$, respectively). Therefore, we equate the values of the two last terms. It results in characteristic magnetic fields where $b_{0} \approx B_{0}$. This estimate shows that the balance between the enhancement and the weakening of the magnetic field fluctuations is achieved in two steps. The main balance is fulfilled at the level of adjusting the turbulent motions without inclusion of the ohmic dissipation. The remaining small unbalance is of the order $\eta / u_{0} l_{0}$ of the main energy level and is eliminated by the ohmic diffusion. In the case $t_{\eta} \ll t_{\beta}$ (the case of a local stationary and homogeneous field), the first and third terms are of the same order, but this does not change the estimation. We note that this estimation is also correct for two-dimensional (2D) turbulence.

Finally, we shall obtain the estimation of magnetic fluctuations for the stationary state directly from Eq. (35). In this case, we have to equate the terms $\gamma B_{0}^{2}$ and $\eta\left\langle(\nabla \times \boldsymbol{b})^{2}\right\rangle$. This yields $\left(b_{0} / B_{0}\right)^{2} \approx\left(\gamma l_{0}^{2} / \eta\right)$. The expression $\gamma B_{0}^{2}$ arises from $\left\langle\boldsymbol{b} \cdot\left(\nabla \times\left(\boldsymbol{u} \times \boldsymbol{B}_{0}\right)\right)\right\rangle$ for permanent $\boldsymbol{B}_{0}$. This gives the relation $\gamma \sim\left(b_{0} / B_{0}\right)\left(u_{0} / l_{0}\right)$. In this way we obtain the final estimate $b_{0} \sim\left(u_{0} l_{0} / \eta\right) B_{0}$. For the case $t_{\eta} \ll t_{\beta}$ this estimate coincides with that given above. Strictly speaking, the opposite case $l_{0} \sim R_{0}$ is not internally consistent. In this case, the difference between fluctuations and the mean magnetic field disappears. Possibly, in this case, our simple two-scale model of turbulence fails.

\section{The case of two-dimensional turbulence}

The paper of Zeldovich (1957) discusses the possibility of very large magnetic fluctuations in turbulent media, particularly the case of 2D-turbulence. The equations for $A_{0}^{2}$ and $\left\langle a^{2}\right\rangle$ in this case are:

$$
\frac{\partial A_{0}^{2}}{\partial t}=-2 \eta B_{0}^{2}+2 A_{0} F_{0}+\eta \nabla^{2} A_{0}^{2}
$$

$$
\begin{aligned}
\frac{\partial\left\langle a^{2}\right\rangle}{\partial t}= & -2 \eta\left\langle b^{2}\right\rangle-2 A_{0} F_{0} \\
& +\eta \nabla^{2}\left\langle a^{2}\right\rangle-\operatorname{div}\left\langle\boldsymbol{u}\left(2 A_{0} a+a^{2}\right)\right\rangle
\end{aligned}
$$

Here, the vector potential $\boldsymbol{A}=\boldsymbol{e}_{z}\left[A_{0}(x, y, t)+a(x, y, t)\right]$, the magnetic field $\boldsymbol{B} \equiv \boldsymbol{B}_{0}+\boldsymbol{b}$ and turbulent velocity $\boldsymbol{u}$ are perpendicular to the $z$-axis with the unit vector $\boldsymbol{e}_{z}$. All values depend only on the coordinates $x, y$ and the time $t$. The mean electromotive force is directed along the $z$-axis and is equal to $F_{0}=-\operatorname{div}\langle\boldsymbol{u} a\rangle$. In the diffusion approximation one has $F_{0} \simeq \beta \nabla^{2} A_{0}$.
Zeldovich considered only one volume-integrated equation for the $\left\langle A^{2}\right\rangle=A_{0}^{2}+\left\langle a^{2}\right\rangle$ quantity:

$\frac{\mathrm{d}}{\mathrm{d} t} \int \mathrm{d} V\left[A_{0}^{2}+\left\langle a^{2}\right\rangle\right]=-2 \eta \int \mathrm{d} V\left[B_{0}^{2}+\left\langle b^{2}\right\rangle\right]$.

He neglected the term with $\left\langle a^{2}\right\rangle$ on the left-hand side and the $B_{0}^{2}$ term on the right-hand side and set $\mathrm{d} A_{0}^{2} / \mathrm{d} t \approx$ $\beta A_{0}^{2} / L_{0}^{2} \approx \beta B_{0}^{2}$ to arrive at the estimate $\left\langle b^{2}\right\rangle_{\max } \approx \beta / \eta B_{0}^{2}$ ( $B_{0}$ is the initial magnetic field).

Physically, this procedure corresponds to a law of conservation - the decrease in $A_{0}^{2}$ is equal to the dissipation of fluctuations due to the ohmic losses. Equations (41) and (42) show that the integral amount of $\left\langle A^{2}\right\rangle$ is conserved if one neglects the terms with the ohmic diffusivity $\eta$. Zeldovich did not neglect this molecular diffusivity. Thus, his estimate is inconsistent ab initio. To see this more clearly, we write the Eqs. (41) and (42) in diffusion approximation:

$\frac{\partial A_{0}^{2}}{\partial t}=-2(\eta+\beta) B_{0}^{2}$

$\frac{\partial\left\langle a^{2}\right\rangle}{\partial t}=-2 \eta\left\langle b^{2}\right\rangle+2 \beta B_{0}^{2}$

In these equations we have omitted the terms with $\nabla^{2}$ and div which do not give the integral contribution. Thus, the evolution of $A_{0}^{2}$ does not alter with fluctuations, as was accepted by Zeldovich. The value $A_{0}^{2}$ monotonically decreases with the characteristic time of turbulent mixing $t_{\beta} \approx L_{0}^{2} / \beta$. The mean-square fluctuation $\left\langle a^{2}\right\rangle$ increases from the initial zero value with the same characteristic time $t_{\beta}$, reaches its maximum value at the time $t_{a}$ and then tends to zero with the characteristic time $t_{\eta} \approx l_{0}^{2} / \eta$. The system eventually reaches the stationary state, for which both the mean magnetic field and its fluctuations disappear, in accordance with the Zeldovich dynamo-suppression theorem. According to these equations, the source of magnetic fluctuations is the turbulent mixing of the initial magnetic field. Because $t_{\eta} \gg t_{\beta}$, the level of $A_{0}^{2}$ tends to zero more rapidly then that of $\left\langle a^{2}\right\rangle$. This means that for $t>t_{\beta}$, the level of magnetic field fluctuations increases relative to the mean magnetic field.

Neglecting the term $\partial\left\langle a^{2}\right\rangle / \partial t$ in (42) implies Eq. (45) with a zero left-hand side, i.e. the Zeldovich estimation gives the relative level of magnetic fluctuations just for the moment $t_{a}$ of the maximum of $\left\langle a^{2}\right\rangle$ :

$\left\langle b^{2}\left(t_{a}\right)\right\rangle \approx \beta / \eta B_{0}^{2}\left(t_{a}\right) \approx R_{\mathrm{m}} B_{0}^{2}\left(t_{a}\right)$.

Here, $R_{\mathrm{m}}$ is the magnetic Reynolds number.

The estimate (46) relates the magnetic fluctuations $\left\langle b^{2}\right\rangle$ to the mean (not the initial!) magnetic field at the instant when the potential fluctuations reach a maximum. The time $t_{b}$ corresponding to the maximum of $\left\langle b^{2}\right\rangle$ does not coincide with the time $t_{a}$. It seems $t_{a}>t_{b}$ because the magnetic fluctuations $\boldsymbol{b}$ are represented by spatial derivatives of the potential $\boldsymbol{a}$, i.e. they are more complex compared to the latter and are earlier influenced by the 
ohmic dissipation. We note that our previous estimate was $\left\langle b^{2}\left(t_{b}\right)\right\rangle \approx B_{0}^{2}\left(t_{b}\right)$.

The characteristic time $t_{\eta} \approx l_{0}^{2} / \eta$ depends strongly on the characteristic space-scale $l_{0}$, which in turn depends on the particular spectrum of the turbulence. For the spectra with slow decrease (for example, the Kolmogorov spectrum with the $p^{-5 / 3}$ - decrease) the parameter $l_{0}$ is near the scale of the dissipation and the value $u_{0} l_{0} \sim \eta$. For the spectra with a sharper decrease, $l_{0} \sim R_{0}$. In the first case $t_{a} \approx t_{b}$, and for the second case these times may be separated considerably.

Nevertheless, the estimate (46) is invalid. The diffusion approximation $F_{0} \equiv-\operatorname{div}\langle\boldsymbol{u} a\rangle \simeq \beta \nabla^{2} A_{0}$ itself implies the relationship between the turbulent diffusivity and all involved values: $\beta \approx u_{0} l_{0} b_{0} / B_{0}$, where $b_{0}^{2}=\left\langle b^{2}\right\rangle$. The substitution of this estimate into (46) gives the final estimation:

$b_{0}\left(t_{a}\right) \approx\left(u_{0} l_{0} / \eta\right) B_{0}\left(t_{a}\right)$.

(47) follows directly from Eq. (42) without recourse to $\beta$. Strictly speaking, parameter $l_{0}$ depends on the time and in (47) we must take its value at the moment $t_{a}$. The estimation (47) confirms our scenario of the evolution of the magnetic field fluctuations described above. For the case $u_{0} l_{0} \sim \eta$, when $t_{a} \approx t_{b}$, this estimation gives $b_{0}\left(t_{b}\right) \approx B_{0}\left(t_{b}\right)$, which was earlier obtained from Eq. (40). For the case of turbulent spectra with a sharp decrease, expression (47) gives very high relative levels of magnetic fluctuations compared to very small value of mean magnetic field $B_{0}\left(t_{a}\right)$. This demonstrates that it is more convenient to make the estimations directly from exact equations, as in the 3D-case.

The diffusion approximation cannot be used in determining the stationary state. In the 2D-turbulent motions we have $\operatorname{div} \boldsymbol{E}_{0} \equiv 0$ and the steady-state condition $\nabla \times \boldsymbol{E}_{0}=0$ means that $\boldsymbol{E}_{0}=0$, i.e. $F_{0}=-\eta \nabla^{2} A_{0}$. Substitution of this expression into (42) and volume integration gives:

$\int \mathrm{d} V\left[B_{0}^{2}+\left\langle b^{2}\right\rangle\right]=0$.

This means that at the 2D-stationary state $\boldsymbol{B}_{0}=$ 0 and $\boldsymbol{b}=0$, as one would expect according to the Zeldovich dynamo-suppression theorem (1957). The derivation of (48) is a somewhat alternative proof of this theorem.

\section{Suppression of the alpha-coefficient by a large mean magnetic field}

Up to this point, we have analyzed the equations for magnetic fields without the consideration of the NavierStokes equation. The latter contains the Lorentz force, which changes the structure of turbulent motions if the magnetic energy is comparable with the kinetic energy of a fluid. Our estimates are not restricted by the case of small magnetic fields. The parameters of the turbulence $u_{0}, R_{0}, l_{0}$ and $\tau_{0}$ are the real parameters, i.e. they take into account the back-reaction of strong magnetic fields. However it is impossible to know their dependence on the magnetic field without the consideration of a coupled system of the induction Eq. (1) and the Navier-Stokes equation. Due to their complexity, one usually considers these equation in a linearized approximation (see Rüdiger \& Kichatinov 1993; Kichatinov et al. 1994; Gruzinov \& Diamond 1994; Vainshtein 1998). The linear approximation has very restricted validity. The third nonlinear term in Eq. (40) is not small compared to the second, and this is why the linear approximation is invalid in the most cases. Nevertheless, this approximation may serve to describe qualitatively the back reaction of magnetic fields onto the turbulence itself.

Gruzinov \& Diamond (1994) have developed a simple approximate theory of the suppression of the $\alpha$-coefficient, which takes into account the back reaction of the mean magnetic field onto the turbulent motions. They considered the case of a homogeneous mean magnetic field $\boldsymbol{B}_{0}$. The time differentiation of the mean electromotive force $\boldsymbol{F}_{0}=\langle\boldsymbol{u} \times \boldsymbol{b}\rangle=\alpha \boldsymbol{B}_{0}$ then gives rise to the relation:

$\dot{\alpha} \boldsymbol{B}_{0}=\langle\dot{\boldsymbol{u}} \times \boldsymbol{b}\rangle+\langle\boldsymbol{u} \times \dot{\boldsymbol{b}}\rangle$.

Using the approximate linearized expressions $\dot{\boldsymbol{u}} \approx\left(\boldsymbol{B}_{0}\right.$. $\nabla) \boldsymbol{b} / 4 \pi \rho$ and $\dot{\boldsymbol{b}} \approx\left(\boldsymbol{B}_{0} \cdot \nabla\right) \boldsymbol{u}$, they obtained the equation:

$\dot{\alpha}=-\frac{1}{3}\langle\boldsymbol{u} \cdot \nabla \times \boldsymbol{u}\rangle+\langle\boldsymbol{b} \cdot \nabla \times \boldsymbol{b}\rangle / 12 \pi \rho$.

This equation gives the estimation:

$\alpha=-\frac{1}{3} \tau\langle\boldsymbol{u} \cdot \nabla \times \boldsymbol{u}\rangle+\tau\langle\boldsymbol{b} \cdot \nabla \times \boldsymbol{b}\rangle / 12 \pi \rho$,

where $\rho$ is the density of a fluid, and $\tau$ is some characteristic time. The first term in (51) is the usual expression of the $\alpha$-coefficient in a quasi-linear approximation, denoted by $\alpha_{0}$. Using relation (9), which formally follows from (12) for stationary and homogeneous turbulence, we obtain the final formula of Gruzinov \& Diamond:

$\alpha=\alpha_{0} /\left(1+\tau B_{0}^{2} / 12 \pi \rho \eta\right) \approx \alpha_{0} /\left(1+R_{\mathrm{m}} B_{0}^{2} / 12 \pi \rho u_{0}^{2}\right)$.

Here, $R_{\mathrm{m}}=u_{0} R_{0} / \eta$ is the magnetic Reynolds number. According to this formula we obtain very small values of the $\alpha$-coefficient, even for a very small mean magnetic field $B_{0}^{2} / 12 \pi \rho u_{0}^{2} \leq 1 / R_{\mathrm{m}} \approx 10^{-9}-10^{-14}$. More sophistical numerical calculations of Kitchatinov et al. (1994) do not confirm this result. The origin of this disagreement lies in use of the relation (9) and the very large value of the characteristic time $\tau$. The authors have taken $\tau \approx R_{0} / u_{0}$.

Now we shall demonstrate that the expression (52) in reality does not result in such huge decreases in the $\alpha$-coefficient. Indeed, the linear approximation $\left(\dot{\boldsymbol{u}} \approx\left(\boldsymbol{B}_{0} \cdot \nabla\right) \boldsymbol{b} / 4 \pi \rho\right.$ and $\left.\dot{\boldsymbol{b}} \approx\left(\boldsymbol{B}_{0} \cdot \nabla\right) \boldsymbol{u}\right)$ requires the use of the smallest characteristic time and the condition $b_{0} \ll B_{0}$. Relation (9) requires at least a local stationary and homogeneous situation when the condition $b_{0} \ll B_{0}$ does not hold. In the best case, we have $b_{0} \approx B_{0}$ for the spectra of turbulence with a slow decrease, when $u_{0} l_{0} \sim \eta$. Thus, according to our previous estimates, we can take 
$\tau \approx t_{\eta}$. Substituting the characteristic time $t_{\eta} \approx l_{0}^{2} / \eta$ into (52), we obtain:

$\alpha \approx \alpha_{0} /\left[1+\left(u_{0} l_{0} / \eta\right)^{2} B_{0}^{2} / 12 \pi \rho u_{0}^{2}\right]$.

Here $u_{0} l_{0} / \eta \approx 1$. Our formula (53) qualitatively corresponds to the results of calculations by Kitchatinov et al. (1994). Thus, the real decrease in the $\alpha$-coefficient is not so drastically large as seen by Gruzinov \& Diamond (1994).

The advantage of this qualitative approach is its simplicity.

\section{Conclusion}

Various estimations of the magnetic field fluctuations in turbulent media demonstrate that the absolute level of magnetic fluctuations is not large. For the stationary state and at the moment of the maximum fluctuation, $\left\langle b^{2}\right\rangle \approx$ $B_{0}^{2}$. For locally stationary and homogeneous turbulence this estimate is also valid.

The relatively large level of magnetic fluctuation relative to the mean magnetic field occurs only for turbulent spectra with a fast decrease in the inertial range of wave numbers. This occurs in the nonstationary case as a consequence of different laws governing the evolution of these components of the magnetic field. The mean magnetic field decreases according to the turbulent mixing characteristic time, and the fluctuations tend to zero more slowly according to the ohmic absorption time.

The characteristic time of the establishment of a quasistationary regime for the case of turbulent spectra with the slow (as in the Kolmogorov spectrum) decrease in the inertial range of wave numbers is of the order $t_{\eta} \approx l_{0}^{2} / \eta$, when the estimate $u_{0} l_{0} \sim \eta$ applies. For these spectra, we obtained the estimates $b_{0} \approx\left(u_{0} l_{0} / \eta\right) B_{0}$ and $\alpha \approx$ $\left(u_{0} l_{0} / \eta\right) u_{0}$. The exact Seehafer relationship $\boldsymbol{B}_{0} \cdot\langle\boldsymbol{u} \times \boldsymbol{b}\rangle=$ $-\eta\langle\boldsymbol{b} \cdot(\nabla \times \boldsymbol{b})\rangle$ is also valid for the case of locally homogeneous and stationary turbulence. For stationary and inhomogeneous media, we estimate $\alpha=\beta\left\langle\boldsymbol{B}_{0} \cdot\left(\nabla \times \boldsymbol{B}_{0}\right)\right\rangle / B_{0}^{2}$. Thus, the usual $\alpha$-effect does not require a very large level of magnetic fluctuation.

The estimate $\left\langle b^{2}\right\rangle \approx B_{0}^{2}$ also denotes that the back reaction of the magnetic field onto the turbulence must be taken into account for $B_{0}^{2} \sim b_{0}^{2} \geq 4 \pi \rho u_{0}^{2}$.
The improved approximate formula of Gruzinov \& Diamond (1994), taking into account the back reaction of the mean magnetic field onto the turbulent motions, shows a moderate decrease in the $\alpha$-coefficient, qualitatively corresponding to the numerical simulations of Kitchatinov et al. (1994).

The exact integral relationships (16)-(18) can be used as additional criteria for the correctness of numerical simulations of the evolution of a magnetic field approaching the stationary state. It should be noted that the numerical calculations assume frequently the periodic boundary conditions. Our estimations are not applicable to these conditions. Here, we have investigated only the case where the magnetic and electric fields tend to zero beyond the considered volume, conditions which frequently apply for astrophysical objects.

\section{References}

Gruzinov, A. V., \& Diamond, P. H. 1994, Phys. Rev. Lett., 72, 1651

Kitchatinov, L. L., Pipin, V. V., \& Rüdiger, G. 1994, Astron. Nachr., 315, 157

Krause, F., \& Rädler, K.-H. 1980, Mean-field magnetohydrodynamics and dynamo theory (Akademie Verlag, Berlin)

Landau, L. D., \& Lifshitz, E. M. 1960, Electrodynamics of continuous media (Pergamon Press, Oxford)

Moffatt, H. K. 1974, J. Fluid Mech., 65, 1

Moffatt, H. K. 1978, Magnetic field generation in electrically conducting fluids (Cambridge Univ. Press, Cambridge)

Morse, P. M., \& Feshbach, H. 1953, Methods of theoretical physics (McGraw-Hill, New-York), 53

Peckover, R. S., \& Weiss, N. O. 1978, MNRAS, 182, 189

Roberts, P. H. 1967, An introduction to magnetohydrodynamics (Elsevier Publ. Co., New York)

Rüdiger, G., \& Kitchatinov, L. L. 1993, A\&A, 269, 581

Seehafer, N. 1994, Europhys. Lett., 27, 353

Seehafer, N. 1996, Phys. Rev. E., 53, 1283

Silant'ev, N. A. 1997a, Geophys. Astrophys. Fluid Dyn., 84, 99

Silant'ev, N. A. 1997b, JETP, 85, 712

Vainshtein, S. I., \& Rosner, R. 1991, ApJ, 376, 199

Vainshtein, S. I., \& Cattaneo, F. 1992, ApJ, 393, 165

Vainshtein, S. I. 1998, Phys. Rev. Lett., 80, 4879

Weiss, N. O. 1994, in ed. M. R. E. Proctor, \& A. D. Gilbert, Lectures on solar and planetary dynamos (Cambridge Univ. Press, Cambridge)

Zeldovich, Ya. B. 1957, JETP (Sov. Phys.), 4, 460 